\title{
Atabaque: uma contribuição de sucesso na evolução de processos
}

\author{
Viviane Malheiros ${ }^{1,2}$, Serge Rehem ${ }^{2}$, José Carlos Maldonado ${ }^{1}$ \\ ${ }^{1}$ Instituto de Ciências Matemáticas e de Computação - USP \\ Caixa Postal 668 - 13560-970 - São Carlos - SP - Brazil \\ ${ }^{2}$ Serpro - Serviço Federal de Processamento de Dados \\ Av. Luiz Vianna Filho, 2355, Paralela, Salvador/BA, Brazil 49010-000
}

\begin{abstract}
For many software development organizations, having well-known, documented and continuously improved processes can be a business advantage. Process documentation, however, is not a trivial task. The efficient maintenance of process assets, while guarantying its general integrity, can be an onerous, complex and very error prone task. Therefore, it is important to provide tools to support process documentation and evolution. In this article, we present Atabaque, a free and simple solution for processes documentation on a collaborative environment, that is based on open standards as XML, XSL and HTML. After almost two years since its conception and first use, it is possible to analyze some benefits of its use.
\end{abstract}

Resumo. Para muitas organizações de desenvolvimento de software, processos documentados, conhecidos e continuamente melhorados podem ser um grande diferencial. A documentação de um processo, contudo, não é tarefa trivial. A manutenção eficiente desses ativos, incluindo a garantia da integridade geral do processo, pode ser uma atividade muito onerosa, complexa e propensa a erros. Por isso, é importante prover ferramentas para apoiá-la. Nesse artigo, apresentamos Atabaque, uma solução livre e simples para documentação de processos de forma colaborativa, baseada em padrões abertos como XML, XSL e HTML. Há quase dois anos desde sua concepção e primeira utilização, já é possível analisar os benefícios de seu uso.

\section{Introdução}

A definição e evolução de processos de software não são tarefas triviais. Um processo de software bem definido deve indicar as atividades a serem realizadas, os recursos necessários, os produtos e modelos a serem elaborados/ utilizados, os procedimentos a serem executados (métodos, técnicas, etc.), as ferramentas e os papéis a serem desempenhados.

Várias pesquisas destacam a melhoria dos processos de produção de software como uma opção para aumentar a qualidade do produto de software e/ou reduzir o tempo e o custo de desenvolvimento, associando a qualidade do produto a qualidade do processo adotado para produzi-lo. Muitos estudos têm sido conduzidos explorando diferentes perspectivas da melhoria de processos: definição de processos e sua evolução contínua; institucionalização de processos; adequação de processos a diferentes contextos e perfis (ex: adoção de métodos ágeis ou de abordagens tradicionais - Boehm 
and Turner(2004)); padronização x customização de processos; etc. Essas vertentes de pesquisa têm em comum, em menor ou maior grau, a necessidade de documentação (explicitação) de todo o processo ou parte dele.

Neste contexto, a documentação de processos, sua evolução e a sua disponibilização são atividades importantes. Essas atividades viabilizam o compartilhamento e a retenção do conhecimento de uma organização. Por meio do processo é possível saber "quem faz o que", "quando" e "como" para que um objetivo seja atingido. Quanto mais amigável for o acesso ao seu conteúdo, mais fácil será a absorção do conhecimento embutido naquele processo e, conseqüentemente, a inovação e a geração de novo conhecimento a partir do conhecimento explicitado no processo (REHEM, 2006).

Além de definir um processo, para que ele se mantenha adequado, é preciso melhorá-lo. No entanto, a manutenção eficiente do processo e da sua documentação , garantindo a integridade do mesmo, pode ser uma atividade onerosa, complexa e propensa a erros. Osterweil (1997) estabelece uma analogia entre software e processo de software, uma vez que ambos são executáveis, possuem um conjunto de requisitos, apresentam benefícios se modelados por uma variedade de técnicas e evoluem guiados por medições. Essa analogia é interessante para entender a magnitude de manutenções em processos de desenvolvimento de software (MALHEIROS, 2007). Tal qual acontece nas manutenções evolutivas e adaptativas de software, é possível identificar e tratar fraquezas de uma versão de um processo de desenvolvimento, convertendo-as em melhorias para a próxima versão. Nesse contexto, soluções automatizadas que apóiem a documentação e a evolução de processos podem ser muito úteis.

Essa foi a principal motivação para a concepção da ferramenta livre Atabaque, cujo objetivo é contribuir para a documentação e evolução de processos documentados em formato de sistemas hipermídia. Sua premissa básica é separar o conteúdo do processo $\left(\mathrm{XML}^{1}\right)$ da sua forma de apresentação $\left(\mathrm{HTML}^{2}\right)$.

Este trabalho apresenta Atabaque, procurando antes esclarecer os fundamentos para sua compreensão. Após mais de um ano desde sua concepção e primeira apresentação no Conserpro $2006^{3}$ é possível apontar os benefícios e as limitações da solução, bem como a evolução de suas funcionalidades. Além da esperada facilidade de atualização do processo, a possibilidade de controlar e explorar meta-dados do processo tem se mostrado muito interessante. A atividade de adaptação de processos (tailoring) também pode ser otimizada com a utilização da solução. Também chama a atenção o interesse pelo assunto por parte da comunidade de software livre.

$\mathrm{Na}$ Seção 2, são descritos alguns conceitos de melhoria de processo. A ferramenta Atabaque, e sua contribuição para melhoria de processo, é descrita na Seção 3. Os resultados alcançados até o momento e a aplicação da ferramenta em uma organização de desenvolvimento de software de grande porte procuram demonstrar como Atabaque pode contribuir para a superação dos entraves inerentes a evolução dos

1 XML (eXtensible Markup Language) é uma linguagem puramente baseada em texto, criada pelo consórcio $\mathrm{W} 3 \mathrm{C}$, que está rapidamente se tornando um padrão para troca de informações entre sistemas (www.w3.org/XML/).

2 HTML (Hyper Text Markup Language) é uma linguagem de marcação de hiper texto utilizada para construção de páginas Web, visualizadas através de programas chamados navegadores (ou browsers).

3 O Conserpro é um congresso nacional interno promovido desde 2004 pelo Serpro. 
processos (Seção 4). Alguns trabalhos relacionados são comentados na seção 5. Por fim, as conclusões, contribuições e oportunidades de trabalhos futuros são consolidadas na Seção 6.

\section{Conceitos Básicos}

Entende-se por processo de software um conjunto coerente de políticas, estruturas organizacionais, tecnologias, procedimentos e artefatos que são necessários para conceber, desenvolver e manter um produto de software (FUGGETTA, 2000). Um processo de software bem definido deve indicar as atividades a serem executadas, os recursos requiridos, os artefatos consumidos e produzidos e os procedimentos a serem adotados (métodos, técnicas, modelos de documentos, entre outros) (BERTOLLO, SEGRINI e FALBO, 2006).

Os processos de desenvolvimento normalmente se enquadram em duas categorias: Técnicos - são processos orientados ao produto, envolvem a especificação e criação dos produtos resultantes do projeto; e Gerenciais - são processos que envolvem a organização do trabalho dentro do projeto. Independentemente da categoria, é possível identificar os componentes de um processo de software (atividades, artefatos, ferramentas, etc.).

Os processos (e seus componentes) podem ser documentados/disponibilizados de várias formas. $\mathrm{O}$ foco deste trabalho são os processos documentados em formato de sistemas hipermídia. A definição e manutenção de processos são tarefas complexas, inerentemente baseadas em conhecimento e suscetíveis a vários tipos de problemas, agrupados neste artigo em duas categorias: problemas semânticos e problemas de exibição:

Os problemas semânticos são aqueles relacionados com o conteúdo, o significado de cada ativo do processo. A utilização da expressão "problema de conteúdo" será evitada para que não se confunda com o significado atribuído à palavra "conteúdo" na expressão "geração de conteúdo", utilizada neste artigo. São exemplos de problemas semânticos: definição equivocada de termos; fórmulas de cálculo erradas; definição de procedimentos burocráticos e pouco eficientes.

Os problemas de exibição são aqueles relacionados com a forma como os ativos documentados do processo são disponibilizados. Estão nessa categoria erros como: tamanho e tipo de fonte; links quebrados e inconsistentes; alinhamento; cores; e consistência entre a forma de exibição e o conteúdo semântico que está sendo exibido.

Os dois tipos de problemas precisam ser tratados e, paulatinamente, eliminados do processo. Ambos demandam tempo e recurso e podem influenciar negativamente na qualidade do processo e, conseqüentemente, do produto final.

Apesar de serem de mais simples identificação e correção, os problemas de exibição podem comprometer a obtenção de conhecimento a partir do processo de desenvolvimento documentado e promover resistência à sua utilização, além de gerar descrédito no conteúdo disponibilizado. Portanto, pode-se afirmar que problemas de exibição têm impacto na usabilidade do processo.

O interesse em usabilidade cresceu após o gradativo reconhecimento do quanto as interfaces são mal projetadas e do quanto uma interface elegante pode trazer de benefício (SHNEIDERMAN e PLAISANT, 2004). A norma NBR/ISO 9241-11 define usabilidade como a medida na qual um produto pode ser usado por usuários específicos 
para alcançar objetivos específicos com eficácia, eficiência e satisfação em um contexto de uso específico.

As pesquisas na área de processos de software nos últimos anos têm explorado duas principais vertentes (ARBAOUI et al.,2002 apud BERTOLLO, SEGRINI e FALBO, 2006): (i) abordagens para modelagem, análise e melhoria do processo de software; (ii) tecnologia de apoio ao processo de software. A primeira vertente enfoca abordagens e melhores práticas para estruturação, organização, documentação e descrição formal de processos de software e inclui normas de qualidade de processo de software e modelos de qualidade de processo (BERTOLLO, SEGRINI e FALBO, 2006), tais como o CMMI (SEI, 2002) e o MPS.BR (SOFTEX, 2005). O mercado fornece também arcabouços (frameworks) de processos que podem ser reutilizados nas empresas. Um exemplo é o Rational Unified Process - RUP (RUP, 2005). A segunda vertente enfoca desenvolvimento de Ambientes de Desenvolvimento de Software (ADS) Centrado em Processos, integrando ferramentas de apoio ao desenvolvimento de artefatos com ferramentas de apoio à modelagem e execução de processos de software (BERTOLLO, SEGRINI e FALBO, 2006).

Seja baseado em uma ou em outra vertente, atualmente é amplamente reconhecido por pesquisadores que a utilização de métodos, processos e ferramentas de desenvolvimento em projetos pode contribuir decisivamente para a qualidade do produto final. Em ambas as vertentes, uma descrição efetiva dos ativos do processo pode ser decisiva para a compreensão tanto do objetivo de cada componente isoladamente (atividade, papel, etc.) quanto do fluxo a ser adotado (integração dos componentes).

Atabaque pode apoiar a definição/manutenção de processos, (a) agilizando e minimizando a possibilidade de ocorrência de problemas de exibição e (b) possibilitando a exploração de diferentes formas de apresentação, potencialmente contribuindo para a usabilidade do processo.

\section{Atabaque}

Atabaque é uma solução livre e simples para documentação de processos de forma colaborativa, baseada em padrões abertos como XML, XSL e HTML. Sua principal vantagem é permitir que os criadores e mantenedores de processos se preocupem eminentemente com os problemas semânticos (problemas do processo diretamente relacionados com suas atividades, entradas, saída, artefatos, etc.) e não com os problemas de exibição. Adicionalmente, estudos da usabilidade da documentação do processo podem rapidamente evoluir para intervenções na exibição, sem que o conteúdo do processo seja afetado. $\mathrm{O}$ isolamento das duas perspectivas aumenta a flexibilidade da evolução do processo. $\mathrm{O}$ encapsulamento de alguns passos e a geração automática de alguns itens da documentação do processo aumentam a integridade do processo como um todo.

O movimento de software livre vem ganhando espaço no mercado e despertando o interesse da academia, do governo e da indústria. Software Livre é qualquer software cuja licença garanta ao seu usuário liberdades relacionadas ao uso, alteração e redistribuição. Seu aspecto fundamental é o fato do código-fonte estar livremente disponível para ser lido, estudado ou modificado por qualquer pessoa interessada (REIS, 2003). A solução Atabaque, aqui apresentada, está consonante com o movimento de software livre pois foi concebida e disponibilizada como um software 
livre e pode ser lida, estudada ou modificada por qualquer pessoa interessada. Além disso, a ferramenta utiliza componentes livres e gera todas as informações do processo em padrões abertos.

A solução Atabaque é baseada em componentes, permitindo a aplicação das suas funcionalidades como um todo ou o uso de apenas alguns de seus componentes. Além disso, a própria evolução da ferramenta pode acontecer isoladamente. As principais funcionalidades disponíveis em Atabaque são: Componente Editor Visual; Componente Gerador de Site e Processo Modelo.

\subsection{Gerador de Site}

Para representação do conteúdo do processo gerado a partir de Atabaque foi escolhida linguagem XML (BRAY, 2004), por se tratar de um padrão aberto e baseado em arquivos puramente texto, de fácil compreensão. Um processo definido/ mantido em XML é facilmente lido por humanos, convertido para bancos de dados relacionais, transformado para exibição em HTML e integrado com ferramentas visuais de modelagem de processo.

O fato de ser um padrão aberto facilita a manutenção colaborativa de Atabaque e viabiliza, por exemplo, que uma empresa compartilhe seu processo para que outras a tomem como modelo e o evoluam conforme sua conveniência. São inúmeras as possibilidades de compartilhamento de conhecimento que podem ser proporcionadas a partir da abordagem proposta.

A utilização de XML, e sua possível exibição em HTML, tem o benefício de permitir a utilização de browsers para a navegação do processo, o que pode trazer ganhos para usabilidade como:

- facilidade de aprendizado, o processo deve ser fácil de aprender;

- permanência, deve ser fácil lembrar-se de como navegar pelo processo, para que mesmo o usuário eventual possa retomar o seu uso, após longo períodos sem utilizá-lo, sem a necessidade de aprender todos os procedimentos de novo;

- acessibilidade, flexibilidade para a sua utilização por pessoas com necessidades especiais, bem como a utilização em diferentes ambientes e situações, e através de vários equipamentos ou navegadores;

- satisfação, o processo deve ser agradável de usar, agradando subjetivamente aos usuários.

Por essas razões, e por ser um padrão altamente difundido na internet, o formato de apresentação adotado é o HTML, permitindo a visualização do processo usando qualquer navegador, independente de sistema operacional.

O componente Gerador do Site é responsável pela transformação de XML para HTML por meio da linguagem XSL - eXtensible Stylesheet Language (ADLER, 2001). XSL também é um padrão aberto baseado em XML, criado com o propósito de processar toda estrutura hierárquica de um documento XML gerando uma saída normalmente em formato texto, HTML ou até mesmo outro XML. De forma simplificada, um arquivo XSL define as regras de transformação de um documento XML. Detalhes sobre o funcionamento desse componente estão disponíveis em atabaque.sourceforge.net . Na Figura 1, é ilustrado um exemplo de sítio de processo construído com a ferramenta Atabaque, resultado da aplicação do componente Gerador 
do Site.

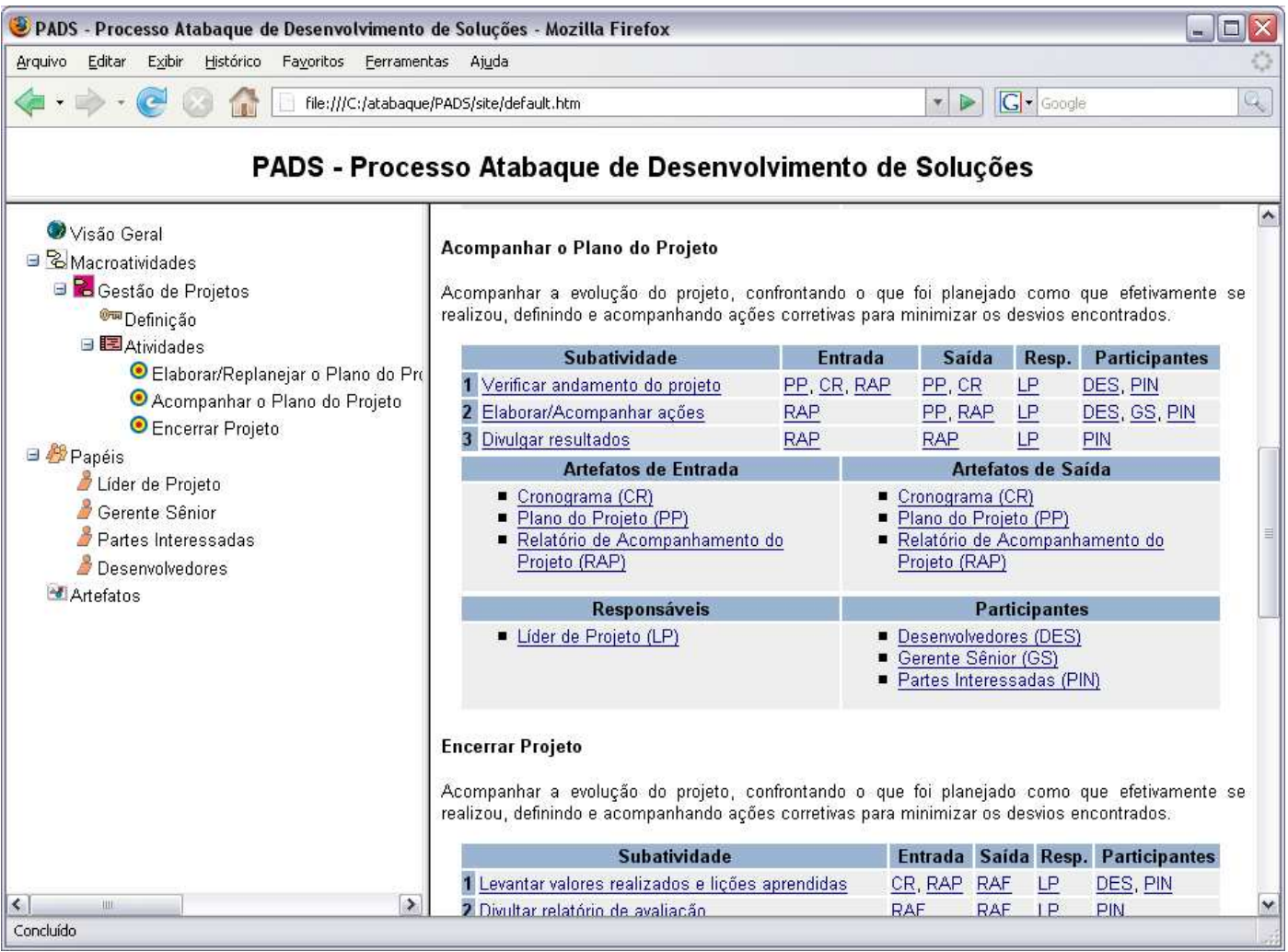

Figura 1 - Exemplo de sítio de processo gerado com Atabaque

\subsection{Editor Visual}

Sem o uso da ferramenta Atabaque, qualquer processo poderia ser totalmente construído utilizando-se um editor de textos comum para editar os arquivos XML e XSL. Para gerar os arquivos HTML, a partir dos arquivos XML e XSL, seria necessário utilizar algum processador (parser) XML para promover as transformações especificadas (o Gerador de Site utiliza um parser desse tipo). O conjunto de todos arquivos HTML gerados seriam disponibilizados em um servidor Web e formariam o sítio do processo.

No entanto, o acompanhamento de sucessivas manutenções em processos documentados em HTML mostrou que essa atividade pode demandar muito esforço e limitar o perfil das pessoas que atuam diretamente na evolução de um processo, já que conhecer HTML torna-se um habilidade requerida. Embora seja mais fácil editar o XML de um processo (pois não é necessário preocupar-se com formatação, apenas conteúdo), ainda assim seria necessário alguma compreensão das regras exigidas pelo XML. Nesse contexto, editores visuais que facilitem o trabalho de construção do processo, isolando o mantenedor do processo de detalhes técnicos da implementação em XML, podem ser muito úteis. Um componente Editor Visual foi desenvolvido para Atabaque, eliminando a necessidade de utilização de editores de texto ou de HTML/XML.

O editor visual (Figura 2), além de permitir a edição de todo o conteúdo do processo, permite o acionamento do componente Gerador de Site para executar as transformações definidas no XSL. 


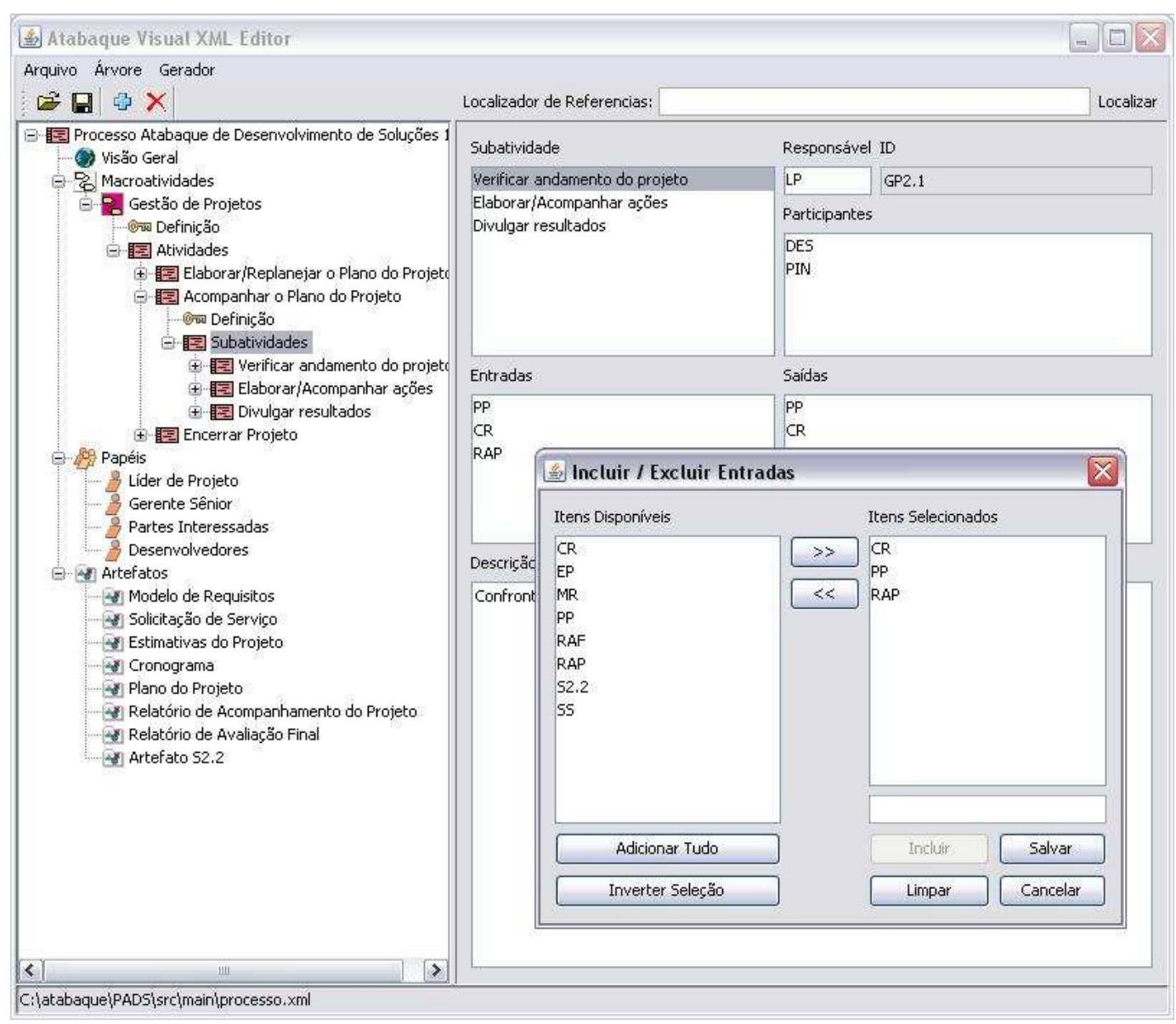

Figura 2 - Editando o conteúdo de um processo com Atabaque

A partir da Figura 2, é possível perceber como a manutenção do processo acontece de forma isolada dos detalhes técnicos de documentação. $\mathrm{O}$ editor traz uma árvore com todos os componentes do processo, organizados de acordo com a estrutura estabelecida para o processo (ver exemplo na seção 3.3 Processo Modelo). Um mantenedor de processo pode, então, selecionar um componente do processo para alteração, criar novos componentes ou excluí-los. No exemplo, o mantenedor está trabalhando em uma sub-atividade ("Verificar Andamento do Projeto"), podendo alterar sua descrição ou qualquer atributo, como participantes, entradas e saídas. Vale ressaltar que nesse momento, nenhuma característica visual está sendo trabalhada. O mantenedor do processo está focado apenas no seu conteúdo. Apenas, poucos recursos de edição de texto estão disponíveis basicamente para permitir ênfase em trechos, como alteração de efeitos de fonte para negrito, itálico ou sublinhado. Para tal, é utilizada a notação Wiki ${ }^{4}$, auxiliada por um componente criado para este fim (Figura 3). O usuário também não precisa conhecer a notação Wiki já que botões para formatação de texto são disponibilizados (Figura 3). A limitação dos recursos disponíveis de edição de texto nesse editor é proposital, já que a intenção é que o foco seja eminentemente no conteúdo. As características visuais do processo serão trabalhadas isoladamente em arquivos XSL e folhas de estilo $\mathrm{CSS}^{5}$, por pessoas com perfil para essa atividade. Esse

4 A notação Wiki possui uma sintaxe simples baseada em texto (por exemplo, usar "__texto__" para exibir um trecho em negrito.

5 Cascading Style Sheets é uma linguagem de estilo utilizada para definir a apresentação de documentos escritos em uma linguagem de marcação, como HTML ou XML. 
isolamento tem o benefício de garantir a padronização visual do processo.

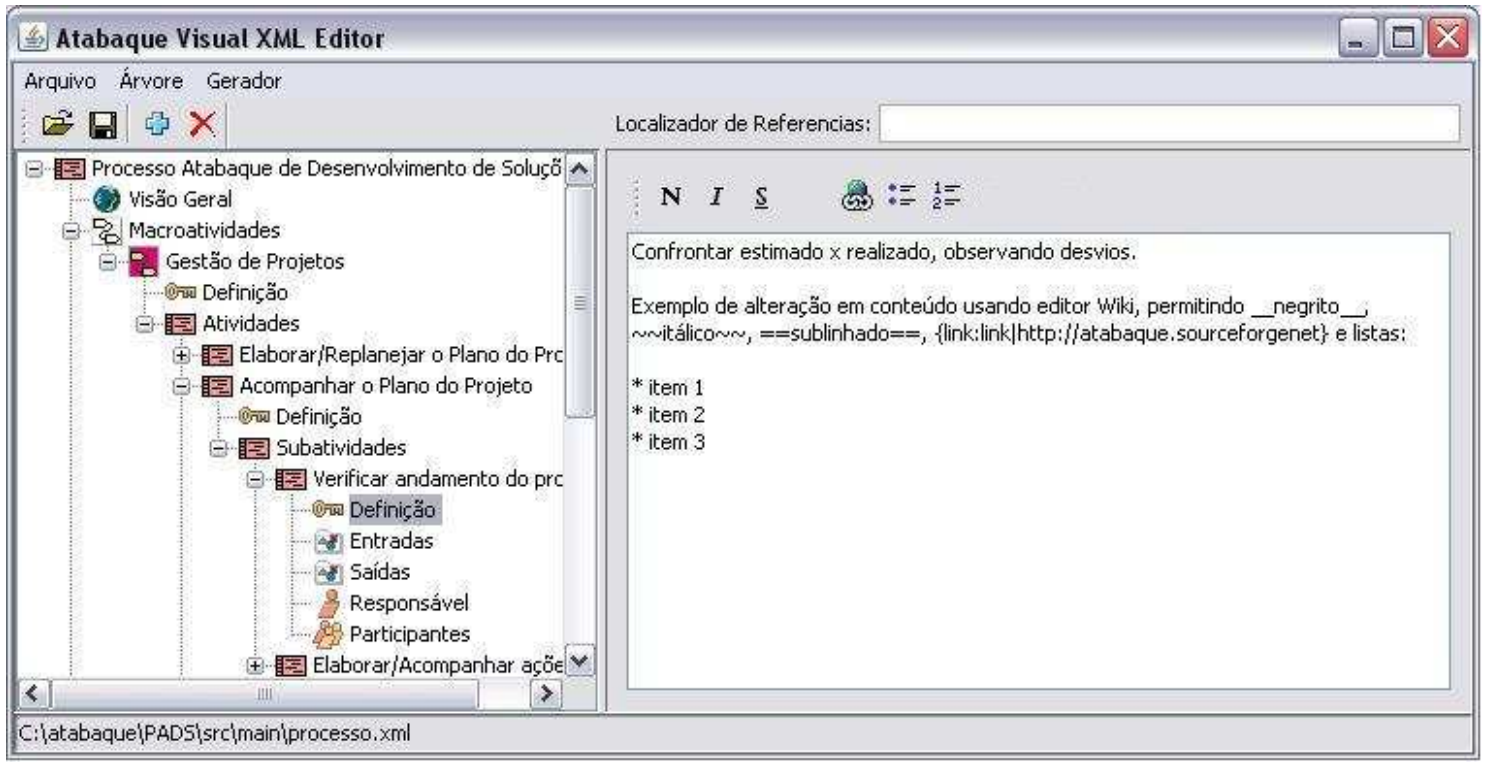

Figura 3 - Usando a notação Wiki para editar conteúdo

\subsection{Processo Modelo}

Para iniciar o uso do Atabaque é preciso, primeiramente, definir o Processo (que está sendo documentado), sua estrutura e componentes (ex: atividade, artefato, papel). Definido o processo conceitualmente, três passos podem ser aplicados para documentação desse processo com o apoio de Atabaque:

1. um mapeamento dos meta-dados do processo (estrutura e componentes) e sua organização em arquivos XML, de acordo com uma estrutura de diretórios específica;

2. a definição das características visuais (folhas de estilo, padrões/tamanhos/cores de fonte, layout do sítio) do processo. Nesse caso, as regras de transformação seriam montadas, por meio da criação de arquivos XSL específicos a cada área de conhecimento;

3. criação de editores visuais específicos para evitar a manipulação direta de XML pelos mantenedores do processo.

A versão de Atabaque disponível no SourceForge (atabaque.sourceforge.net) fornece um processo hipotético (com toda estrutura mapeada e definição de características visuais), que pode ser usado como ponto de partida para o mapeamento de novos processos. Esse processo hipotético foi encapsulado no componente Processo Modelo. O uso do Processo Modelo pode eliminar ou minimizar a necessidade dos passos 1, 2 e 3. A estrutura utilizada pelo componente está representada na Figura 4. Aparentemente, essa estrutura de processo é genérica e pode ser utilizada para a documentação da maioria dos processos de desenvolvimento de software, mas essa adequação precisa ser avaliada e comparada com ontologias de processo existentes. Essa estrutura é compatível com a estrutura do framework RUP (RUP, 2005). 

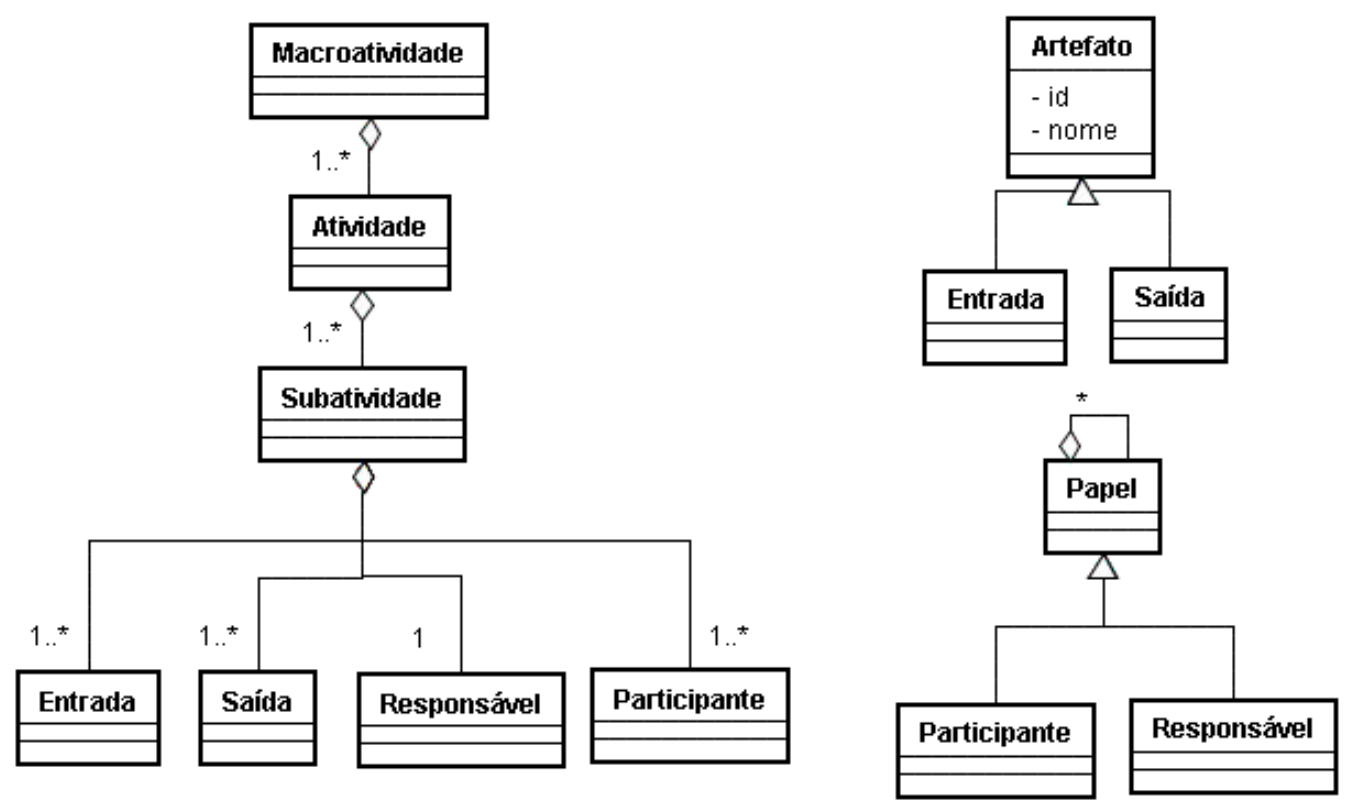

Figura 4 - Estrutura de processo implementada pelo componente Processo Modelo. Diagrama UML adaptado. Um mesmo papel pode ser participante e responsável para atividades distintas. Um mesmo artefato pode atuar como entrada e também como saída.

Caso a estrutura de referência do Processo Modelo (Figura 4) seja equivalente a estrutura definida para o processo a ser documentado, o passo 3 pode ser completamente eliminado com a utilização do componente Editor Visual já disponível em Atabaque. O passo 1, por sua vez, será resumido ao detalhamento dos atributos de cada componente do processo. Caso as características visuais do Processo Modelo sejam adequadas para a organização, o passo 2 pode ser completamente eliminado.

Da forma como foi concebido, Atabaque também pode ser utilizado para "redocumentar" processos existentes em formato hipermídia. Sua estrutura flexível e adaptável (Atabaque se adapta a qualquer processo) permite manter o mesmo resultado visual do processo já documentado e, ao mesmo tempo, melhorá-lo para que se beneficie dos ganhos decorrentes da separação do conteúdo da forma de apresentação. A execução dos passos 1, 2 e 3, especialmente do 2, demandará maior ou menor esforço a depender das diferenças do processo sendo "redocumentado" para o Processo Modelo disponível em Atabaque. Mas, uma vez executado o processo de migração, a evolução do processo poderá se beneficiar de todos os ganhos identificados com o uso da ferramenta Atabaque.

\section{Resultados alcançados}

Logo após a sua criação, a ferramenta Atabaque foi aplicada em um estudo de caso, para sua validação e identificação de oportunidades de melhoria. Observações identificadas à época foram registradas em Rehem (2006).

A divulgação da ferramenta permitiu que os autores tivessem contato com a abordagem de desenvolvimento colaborativo do software livre. Interessados na solução apareceram em alguns lugares do país, um deles tornando-se um desenvolvedor ativo da ferramenta. Em função de comentários recebidos, o editor visual da ferramenta foi melhorado e uma nova versão foi disponibilizada. $O$ interesse na ferramenta pode ser demonstrado pelo histórico de download: entre a ferramenta e sua documentação foram 
mais de 980 downloads (Figura 5), até o meados de março de 2008. É importante ressalvar que, apesar de demonstrar interesse, o histórico de downloads não demonstra satisfação dos usuários ou uso efetivo.

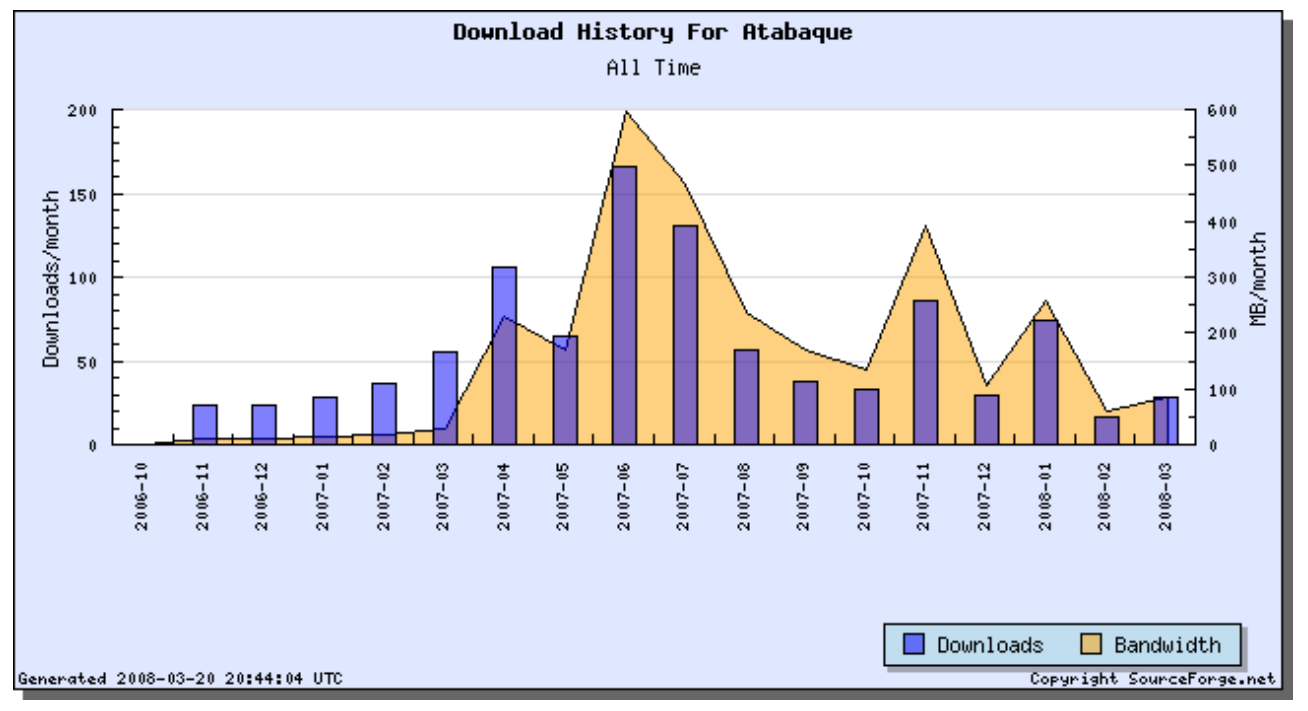

Figura 5 - Histórico da evolução dos downloads em sourceforge.atabaque.net

Várias mensagens eletrônicas foram enviadas para os autores solicitando informações acerca da ferramenta. Observou-se que a proposta notadamente teve ressonância na comunidade de software livre. A simplicidade da ferramenta, o fato desta estar consonante com os princípios de software livre e a possibilidade de apoiar a documentação de qualquer processo, inclusive os inspirados nos princípios ágeis, contribuíram para aumentar o interesse. Também entraram em contato indivíduos da academia e a integração de Atabaque com a uma ferramenta acadêmica de adaptação de processos, Protto (PEREIRA, 2007), começou a ser estudada. Adicionalmente, a ferramenta está sendo avaliada para a definição e manutenção de processos de Gestão de Projetos em uma agência nacional do governo brasileiro. $\mathrm{O}$ intercâmbio com representante dessa agência permitiu a identificação de uma funcionalidade desejável: diagramação visual do processo. Discussões para desenvolvimento dessa funcionalidade estão em andamento, também lastreadas em padrões abertos.

A ferramenta Atabaque (com todos os seus componentes) foi adotada para evolução de parte do processo de desenvolvimento padrão de uma organização de desenvolvimento de software de grande porte (mais de 3.000 desenvolvedores de software). O número de propostas de melhoria para o processo relacionada a questões de exibição reduziu bastante. Nas duas últimas versões do processo, publicadas sem Atabaque, $13 \%$ das propostas de melhoria eram relacionadas a questões de exibição. Nas duas primeiras versões do processo publicadas com o uso de Atabaque, esse percentual caiu para $2 \%$. Na última publicação do processo, disponível há mais de 2 meses, nenhum erro de exibição foi reportado até o fechamento deste artigo . Durante a última homologação do processo, nenhum erro de exibição ou inconsistência entre os componentes do processo foi reportado. Dados relativos a homologações de versões anteriores do processo não estavam disponíveis. No entanto, houve significativa melhoria, de acordo com uma análise subjetiva do grupo mantenedor: "[Na etapa de] homologação é onde mais aparece este tipo de erro de exibição, o fato de ter caído a zero melhorou muito a etapa de homologação". 
A manutenção do processo ficou mais rápida e agora é feita pelo próprio grupo mantenedor do processo, já no momento de definição da alteração. Recentemente, um dos grupos mantenedores do processo fez uma evolução no processo que envolveu: a eliminação de 5 artefatos, 4 atividades e 55 sub-atividades; a alteração de 30 subatividades e 7 atividades e a criação de 1 atividade. A manutenção total de $100 \%$ das atividades e $83 \%$ das sub-atividades foi feita em apenas 5 dias úteis. O próprio grupo estima que uma manutenção equivalente, sem o uso de Atabaque, demandaria ao menos 10 dias úteis, ou $100 \%$ a mais de esforço.

Com o uso de Atabaque, o grupo está focado apenas no conteúdo do processo, questões relacionadas a apresentação ou integridade do que é exibido são tratadas implicitamente pela ferramenta. Antes do uso de Atabaque, a produtividade dos especialistas durante alterações do processo era muito inferior(no exemplo acima a produtividade dobrou), devido a preocupações com questões de exibição e necessidade de editar diretamente (ou através de algum software específico) arquivos HTML. O perfil do mantenedor também foi alterado, já que agora conhecer HTML não e mais um pré-requisito. A alteração pode ser feita diretamente pelo responsável pela definição do processo. Nenhum tempo foi investido para tratar questões visuais.

A organização do conteúdo do processo em XML permitiu a exploração de meta-dados do processo. A definição e o acompanhamento de métricas sobre a melhoria de processos se beneficiam desses meta-dados. Esse potencial ainda deve ser mais explorado. Mas, a identificação dos dados da evolução acima citados já foi feita a partir do processo em Atabaque. Análises do tipo "quantidades de erros por versão implantada" podem ser mais precisas se o tamanho do processo é conhecido. Com a ferramenta é mais fácil responder, por exemplo, qual o percentual do processo alterado em uma manutenção. Se um artefato por exemplo é dissociado de uma atividade, a exclusão desse artefato, automaticamente implicará na remoção dos links para esse artefato no processo.

\section{Trabalhos Correlatos}

Existem trabalhos publicados que apontam para o apoio automatizado a documentação e evolução de processos. Alguns deles foram, de alguma forma, testados empiricamente, outros ainda estão em estágio de proposta.

A principal contribuição da ferramenta Atabaque é o fato de ser uma ferramenta livre (e multiplataforma, portanto independente de sistema operacional) podendo ser estudada, alterada e evoluída por qualquer interessado. Pelo que verificamos, as ferramentas existentes não disponibilizaram seu código. Disponibilizada como ferramenta livre, encontramos apenas ferramenta EPF Composer ${ }^{6}$ (HAUMER, 2007), que parece interessante para a definição de processo. Essa ferramenta também privilegia um formato amigável do processo, mas induz seu usuário a usar a estrutura de processos e forma de visualização encontradas no seu processo (não está claro se outros formatos ou estrutura podem ser utilizados). Aparentemente é uma ferramenta mais completa que Atabaque. Por outro lado, pode ser mais difícil entendê-la e usá-la corretamente. Adicionalmente, a ferramenta não está pronta para ser usada por nativos da língua portuguesa. $O$ fato de Atabaque está estruturado em componentes é outro diferencial da solução, assim, ela pode ser aplicada em diferentes contextos. Encontramos na internet, por exemplo depoimento sobre o uso de um componente

6 EPF Composer 
isolado da ferramenta (Atabaque DTDtoBean, em dtdtobean.sourceforge.net) com o comentário "Good work" (bom trabalho).

Trabalhos sobre documentação de processos são apresentados no contexto de ambientes de desenvolvimento de software (ADS) centrados em processo, estando fortemente ligados a esses ambientes. Esse é o caso das ferramentas AssistPro (FALBO, 1998) e Def-Pro (MACHADO, 2000) - relacionadas com a Estação Taba - e da ferramenta de definição de processos do ambiente ODE (RUY, 2003). Essas ferramentas são restritas ao sistemas operacionais Windows® e não disponibilizam o código fonte. Elas compartilham o enfoque em definição de processos e divergem no suporte a especialização e instanciação de processos. Uma vantagem dessas ferramentas em relação a Atabaque é o fato delas incorporarem, de alguma forma, componentes de gestão de conhecimento (lições aprendidas sobre instanciação, tipo de ADS ou características da organização) para a definição dos processos. Por outro lado Atabaque tem um enfoque maior na apresentação final do processo (ganho em usabilidade), com mais recursos para a documentação de ativos gerais do processo. $\mathrm{O}$ formato final do processo é flexível e independente de seu conteúdo e Atabaque não tem qualquer vínculo com ADS, podendo ser utilizado por qualquer organização, mesmo aquelas que não pretendem migrar para um ADS. Atabaque parece mais adequado para documentação de "framework" de processos. A geração de páginas XML/HTML permite que o processo gerado seja acessado por qualquer máquina que possua um browser. Formatos diferentes de exibição podem ser definidos para cada componente (artefato, atividade, papel, etc.) e recursos de edição de texto estão disponíveis de forma a permitir ênfase onde for necessário.

Foi iniciado um estudo de integração de Atabaque com a ferramenta Protto (PEREIRA, 2007), cujo objetivo é apoiar a adaptação de processos. Essas ferramentas são complementares. Com as funcionalidades de Protto e sua verificação de consistência, Atabaque poderá apoiar melhor a adaptação de processos, ao mesmo tempo que complementará Protto permitindo a integração da estrutura do processo com o seu conteúdo.

Atabaque está integrado ao $\mathrm{CVS}^{7}$ (e pode ser usado com qualquer outro sistema de controle de versão), permitindo controle das alterações no processo. O conteúdo do processo armazenado em XML permite que o CVS apresente as diferenças entre duas versões de um mesmo arquivo, ou ainda que pessoas diferentes trabalhem em paralelo em áreas distintas de um mesmo XML (por exemplo, em sub-atividades de uma mesma atividade), efetivando a junção (merge) ao final. Ao se consolidar uma versão oficial do processo também é permitido gerar linhas-de-base (baselines), recurso útil quando se deseja rastreabilidade das alterações. Nem sempre está claro se outras ferramentas possuem esse recurso, que é importante para uma manutenção colaborativa do processo.

Outra linha de trabalho correlato é a construção de ferramentas de Gestão de Conteúdo que têm o mesmo, ou maior, potencial para uma documentação amigável em formato XML/HTML que Atabaque. As vantagens de Atabaque em relação a essas ferramentas são a sua adequação para documentação de processos e a possibilidade de tratar os meta-dados de processo.

7 Concurrent Version System, um popular sistema de controle de versão de código aberto, muito usado pelas comunidades de software livre (http://www.nongnu.org/cvs/). 


\section{Conclusão e trabalhos futuros}

A ferramenta Atabaque se mostrou um instrumento efetivo para a geração de um processo e manipulação de seu conteúdo. Entre os benefícios de utilização da ferramenta foram observados: (a) rápida geração de páginas HTML, uma vez definidos os arquivos XSL e XML; (b) reutilização da estrutura das páginas (XSL) proporcionando agilidade na geração de novas páginas e minimização de problemas de inconsistência no formato das páginas; (c) facilidade de alteração no formato padrão de cada área de conhecimento (elemento do processo), exigindo a manutenção de apenas um arquivo XSL, ao invés de vários arquivos HTML como seria necessário na implementação de processos utilizando HTML(como na organização onde Atabaque foi implantado); (d) redução de erros de exibição (tamanho, tipo e cor de texto, links e tabelas) em função de todas as características de formatação estarem concentradas em um único XSL; (e) possibilidade de manutenção no site por pessoas com um baixo conhecimento da tecnologia Web.

Atabaque tem pode contribuir para a gestão de conteúdo de processos em geral. Para a organização onde foi implantada, além de contribuir para a evolução do processo de desenvolvimento de software, a ferramenta pode ser adaptada para a evolução de outros processos que usam a tecnologia Web. Atabaque já está sendo utilizada gradativamente para a manutenção do processo de desenvolvimento. Sua validade para a manutenção desse processo pôde ser constatada por meio de sua aplicação no processo de Gestão de Projetos de desenvolvimento de software. Três versões do processo de Gestão de Projetos de desenvolvimento de software foram alteradas com Atabaque. A primeira versão alterada não utilizou o editor visual, que foi utilizado nas duas seguintes.

A possibilidade de convivência pacífica entre partes do processo (as geradas de forma tradicional - edição de HTML - e as mantidas com Atabaque) é outra vantagem, pois pode-se definir um cronograma de plano de implantação, inicialmente focado nas áreas de conhecimento mais críticas, até que todo o processo seja migrado.

Para os autores a experiência de desenvolver, disponibilizar e acompanhar o desenvolvimento de uma solução utilizando a filosofia do desenvolvimento em software livre tem sido enriquecedora, especialmente no que tange a colaboração proporcionada por esse tipo de desenvolvimento

Sendo uma ferramenta livre, Atabaque surge como contribuição para as comunidades de software livre e de engenharia de software. Modelos de processo por área (engenharia de software, gestão de projetos, gerenciamento de serviços de TI, etc.) podem ser facilmente criados e compartilhados. Processos inteiros podem ser disponibilizados e diversos padrões de exibição HTML poderão surgir. Ao invés de algo restrito a poucas pessoas a modelagem de processos com Atabaque pode ser tratada por empresas de diferentes naturezas e tamanhos, envolvendo profissionais de diversas áreas, não necessariamente relacionadas a TIC. Com o aprendizado em diferentes contextos, a ferramenta pode ser evoluída por qualquer interessado e retornar para a comunidade como uma solução mais robusta e efetiva. A integração de Atabaque com outras ferramentas (RUY, 2003; MACHADO, FALBO, 1998) pode ser interessante, já que aparentemente são complementares. O ambiente ODES, apesar de não ter seu código disponível, é desenvolvido em plataforma livre, isso deve facilitar a integração. Atabaque e sua documentação estão disponíveis em http://sourceforge.net/projets/atabaque. 


\section{Referências}

ADLER, Sharon et al. Extensible Stylesheet Languagem (XSL) Version 1.0. World Wide Web Consortium Recomendation, 15 out. 2001. Disponível em: <http://www.w3.org/TR/xsl>. Acesso em: 27 jun. 2006.

ARBAOUI, S. DERMIANE,J., OQUENDO, F.Comparative review of process centered Software Engineering Enviroments". Software Engineering 14, p.311-340.

BERTOLLO, G. SEGRINI, B. FALBO, R. Definição de processos de software em um Ambiente de Desenvolvimetno de Software Baseado em Ontologias. In:V Simpósio Brasileiro de Qualidade de Software, Anais, 2006.

BOEHM, B. and TURNER, R. Balancing Agility and Discipline: A Guide for the Perplexed. Addison-Wesley. Boston, MA, USA, 2004.

BRAY, Tim et al. Extensible Markup Language (XML) 1.0 (Second Edition). World Wide Web Consortium Recommendation, 4 fev. 2004. Disponível em: <http://www.w3.org/TR/REC-xml>. Acesso em: 29 jun. 2006.

FALBO, R. Integração de Conhecimento em um Ambiente de Desenvolvimento de Software. Tese de doutorado, COPPE, UFRJ. Dezembro,1998.

FUGGETTA, A. Software Process: A Roadmap. In: The Future of Software Engineering, ICSE 2000, Ireland, 2000.

HAUMER, P. Eclipse Framework Composer. Available at: http://www.eclipse.org/epf/general/EPFComposerOverviewPart1.pdf. April, 2007.

MACHADO, L. SANTOS, G., OLIVEIRA, K., ROCHA, A.R. Def-pro:Uma ferramenta para apoiar a Definição de Processos Padrões. XI conferência Internacional de Tecnologia de Software (CITS), 2000.

MALHEIROS, V. Malheiros, V. Gestão de Conhecimento para Definição e Melhoria de Processos de Teste. Monografia de Qualificação de doutorado. Orientador: Prof. Dr. José Carlos Maldonado. Universidade São Paulo, abril, 2007.

REHEM, S. Atabaque: Uma ferramenta livre para modelagem de processo de forma cooperativa. Congresso Serpro de Tecnologia e Gestão Aplicadas 2006 (ConSerpro 2006). Salvador, 2006.

OSTERWEIL, J. Software processes are software too (revised). In Proc. Of the 1997 International Conference on Software Engineering, Boston, USA, ACM Press, 1997.

PEREIRA, E. BASTOS, R, OLIVEIRA, T. A Systematic Approach to Process Tailoring. Systems Engineering and Modeling, 2007. ICSEM '07. Israel. Março, 2007.

REIS, C.. Caracterização de um Processo de Software para Projetos de Software Livre.. Dissertação (Mestrado) - ICMC, Universidade São Paulo, São Carlos, 2003. Disponível em: http://async.com.br/ kiko/dissert_usp.pdf. Acesso em abril 2006.

RUP - IBM Rational Unified Process. "Rational Unified Process". Disponível em: http://www-306.ibm.com/software/awdtools/rup/ . Acesso em julho 2005.

RUY, F. BERTOLLO, G., FALBO, R. Apoio Baseado em Conhecimento à Integração de Processo em ODE. JIISIC, 2003 